\title{
Can anti-bothropstoxin-I antibodies discriminate between Bothrops jararaca and Bothrops jararacussu venoms?
}

Ricardo Teixeira Araujo 1,2, Carlos Corrêa-Netto 1,2, Leonora Brazil-Más², Caio Raony Farina Silveira², Irene Fernandes ${ }^{3}$ and Russolina Benedeta Zingali ${ }^{*}$

\begin{abstract}
Background: Snakes of the genus Bothrops, popularly known as pit vipers, are responsible for most cases of snakebite in Brazil. Within this genus, Bothrops jararacussu and B. jararaca deserve special attention due to the severity of their bites and for inhabiting densely populated areas. Regarding the treatment of snakebites by Bothrops jararacussu, questions have been raised about the effectiveness of the specific bothropic antivenom in neutralizing myotoxic effects; however, there are no accurate data for humans. Thus, the development of a differential diagnostic kit for this species would be of great interest because it provides, for healthcare professionals, a tool that would allow us to determine whether the accident was caused by B. jararacussu or other species of the genus. It would also make it possible to evaluate the specificity of the treatment and to provide data for epidemiological studies.
\end{abstract}

Methods: First, we produced a species-specific polyclonal antibody - a potential biomarker of Bothrops jararacussu venom - against bothropstoxin-I (BthTX-I), which is also found in smaller quantities in the venoms of B. jararaca from southern Brazil.

Results: Polyclonal antibodies against bothropstoxin-I could be separated into several species-specific immunoglobulins. Then, aiming to develop a system of safe and standardized immunoassay, we produced monoclonal antibodies. Seven hybridomas were obtained. Five of them were specific to the venom of $B$. jararacussu and two recognized the venom of B. jararaca from the southeastern population. The use of monoclonal antibodies also made it possible to differentiate $B$. jararacussu from B. jararaca venom obtained from the southern population. Analyzing the reactivity of monoclonal antibodies against other bothropic venoms, we found $\mathrm{mAb} B t-3$ to be more specific than others for $B$. jararacussu venom.

Conclusions: These results show the potential of BthTx-I for producing monoclonal antibodies that differentiate between B. jararacussu and other Bothrops species venoms.

Keywords: Bothrops jararacussu, Bothrops jararaca, Bothropstoxin-I, Monoclonal antibodies, Species specificity

\footnotetext{
* Correspondence: Izingali@biogmed.ufrj.br; Izingali@gmail.com

'Laboratório de Hemostase e Venenos, Instituto de Bioquímica Médica

Leopoldo de Meis, Universidade Federal do Rio de Janeiro (UFRJ), Rio de

Janeiro, RJ, Brasil

Full list of author information is available at the end of the article
} 


\section{Background}

Among the snake species of the genus Bothrops, Bothrops jararacussu is remarkable for the low immunogenicity of its venom, which is highly myotoxic, leading to necrosis of striated muscle fibers and slowing tissue regeneration [1, 2]. In all Brazilian states where B. jararacussu is present, B. jararaca is also found [3]. This makes it difficult to differentiate between these two snakes when an accident occurs, due to extensive homology among envenomation symptoms [4, 5]. In Rio de Janeiro, both species have clinical importance. In the envenomation context, the question of the efficiency of specific bothropic antivenom against $B$. jararacussu bites is often discussed, since the myotoxic effects observed in mice are not completely neutralized. For this reason, some researchers have suggested the use of a combinated bothropic-crotalic antivenom as a more appropriate treatment $[1,6-10]$.

The proteomic characterization of $B$. jararacussu venom and the immunoreactivity of anti- $B$. jararaca and anti- $B$. jararacussu sera have contributed to our understanding of some of the immunochemical characteristics of B. jararacussu venom and led to a suggestion for the use of bothropstoxin-I (BthTx-I) as a biomarker molecule [11]. This molecule is a phospholipase $A_{2}$ Lys-49 (Lys-49 $\mathrm{PLA}_{2}$ ) from $B$. jararacussu venom with three $\alpha$-helices and two antiparallel $\beta$-sheets $[12,13]$. This protein is the most abundant myotoxin that can be isolated from $B$. jararacussu venom and although it exhibits severe neurotoxicity and myotoxicity, it shows little or no phospholipase activity $[13,14]$. This toxin is able to promote injury in lipid bilayer of cell membranes through a calciumindependent mechanism, inducing myonecrosis $[13,15]$.

Lys49-PLA $\mathrm{P}_{2}$ molecules have been described in various Bothrops venoms, such as BnSP-7 from B. neuwiedi, myotoxin I from B. moojeni, and BaTX from B. alternatus, among others [16-18]. Recently, Gonçalves-Machado et al. [19] described the presence of BthTx-I in venoms of $B$. jararaca from the southern region of Brazil. Methods for differentiating the envenomation caused by $B$. jararacussu from the one caused by $B$. jararaca are very important in order to enable the epidemiological study of accidents with these two snakes, as well as to allow studies of treatment efficiency for $B$. jararacussu bites in humans. In this context, the objective of the present study was to produce monoclonal antibodies from BthTx-I to be used as tools for the development of a differential diagnostic kit for bites provoked by $B$. jararacussu.

\section{Methods}

\section{Animals and venoms}

$\mathrm{BALB} / \mathrm{c}$ mice $(18-20 \mathrm{~g})$ were provided by the Butantan Institute. All procedures were approved by the Ethics Committee for Animal Research of Butantan Institute (process no. 697/10). The venoms of Bothrops alternatus, $B$. atrox, $B$. diporus, $B$. erytromelas, $B$. fonsecai, B. insularis, B. jararaca, B. jararacussu, B.leucurus, B. moojeni, B. neuwiedi and B. pubescens were provided by the Laboratory of Herpetology of the Vital Brazil Institute.

\section{Isolation of bothropstoxin-I}

Bothropstoxin-I was isolated following the description of Correa-Netto et al. [11]. Briefly, B. jararacussu venom (500 mg) dissolved in $5 \mathrm{~mL}$ buffer $(20 \mathrm{mM}$ Tris- $\mathrm{HCl}$, $150 \mathrm{mM} \mathrm{NaCl}, \mathrm{pH}$ 8.8) was applied onto a Sephacryl S200 HR column $(2.6 \times 100 \mathrm{~cm})$ with a flow rate of $17 \mathrm{~mL} / \mathrm{h}$ generating four distinct peaks. The third peak, which contained proteins with a maximal apparent molecular mass of $30 \mathrm{kDa}$, was dialyzed against PBS buffer (50 mM sodium phosphate/150 $\mathrm{mM}$ sodium chloride, $\mathrm{pH}$ 7.4) and applied to an ion-exchange column (Mono S HR 5/5, Pharmacia) at a flow rate of $1.0 \mathrm{~mL} / \mathrm{min}$. Elution using a linear gradient of $0-1 \mathrm{M} \mathrm{NaCl}$ in the same phosphate buffer yielded two peaks; the second was identified as BthTx-I by mass spectrometry. The elution profile was monitored by absorbance at $280 \mathrm{~nm}$.

\section{Polyclonal anti-BthTx-I serum produced in rabbits}

Two rabbits were injected subcutaneously with $500 \mu \mathrm{g}$ of BthTx-I emulsified in complete Marcol/Montanide adjuvant. After the first injection, boosters were made 2, 3, 4 and 5 weeks later with incomplete Marcol/Montanide adjuvant. Blood samples were drawn after the 5th week and the immune serum was collected.

\section{Purification of polyclonal species-specific antibodies}

Two columns of Sepharose 4B activated by cyanogen bromide were prepared, one with Bothrops jararacussu (jararacussu-Sepharose) and the other with Bothrops jararaca from the southeast region of the country (jararaca-Sepharose). The column preparation followed instructions from Amersham Biosciences. Both columns were equilibrated with PBS buffer $(50 \mathrm{mM}$ sodium phosphate/150 mM sodium chloride, $\mathrm{pH}$ 7.4). Anti-BthTx-I serum was applied to the jararacussu-Sepharose column at a flow rate of $1 \mathrm{~mL} / \mathrm{min}$, thereafter discarding the unbound material. Immunoglobulins with affinity for the $B$. jararacussu venom were collected and applied to the jararaca-Sepharose column at a flow rate of $1 \mathrm{~mL} / \mathrm{min}$. The immunoglobulins that did not bind to the column were collected and those with affinity for the venom of $B$. jararaca were discarded. The pool of IgGs that did not bind to the column was designated "purified speciesspecific anti-BthTx-I". For the elution of immunoglobulins from both columns, $0.2 \mathrm{M}$ glycine $/ \mathrm{HCl}$ buffer, $0.15 \mathrm{M} \mathrm{NaCl}, \mathrm{pH} 2.8$ was used. The elution was monitored by absorbance at $280 \mathrm{~nm}$. 


\section{Immunization of mice}

Mice (female Balb/c) were immunized according to Fernandes et al. [20]. Four days after the last booster, animals were bled from the ophthalmic plexus and one animal immunized with BthTx-I was killed and popliteal lymph nodes were removed.

\section{Production and purification of monoclonal antibodies (mAbs)}

Monoclonal antibodies were produced as described by Köhler and Milstein [21], with modifications. Popliteal lymph node cells from BALB/c mice immunized with BthTx-I were fused with SP2-O cells (2:1) using polyethylene glycol 4000 (Merck). Hybrids were selected in RPMI 1640 medium containing 3\% HAT (hypoxanthine $10 \mathrm{mM}$, aminopterin $40 \mathrm{mM}$ and thymidine $1.6 \mathrm{mM}$; Gibco-BRL) and $10 \%$ FCS (Gibco-BRL) at $37{ }^{\circ} \mathrm{C}$ and $5 \% \mathrm{CO}_{2}$. Supernatant fluids were screened for species-specific antibodies by ELISA, as described in the next section. Antibodysecreting cells were expanded and cloned twice at limiting dilution. The mAbs contained in culture supernatants were purified by affinity chromatography on protein-A Sepharose (Pharmacia) equilibrated in borate saline buffer, $\mathrm{pH}$ 8.5. The proteins were eluted in $0.2 \mathrm{M}$ glycine $/ \mathrm{HCl}$ buffer, $0.15 \mathrm{M} \mathrm{NaCl}, \mathrm{pH} 2.8$, and dialyzed in borate saline buffer. An isotyping kit (Sigma) was used to determine the heavy-chain isotype.

\section{ELISA}

ELISA was carried out according to Theakston et al. [22]. Briefly, wells were coated with venom $(1 \mu \mathrm{g} /$ well $)$ or BthTx-I and, after blocking with $3 \%$ bovine serum albumin, various dilutions of mAbs were added to a final volume of $100 \mu \mathrm{L}$. Antigen-antibody reaction was detected by addition of anti-mouse IgG-peroxidase conjugate and ortho-phenylenediamine (1 mg/mL, Sigma) and $\mathrm{H}_{2} \mathrm{O}_{2}$ as enzyme substrates.

\section{Dot blot}

Dot blot was carried out as described by Towbin et al. [23], with modifications. Briefly, samples $(1 \mu \mathrm{g} / \mu \mathrm{L})$ were applied directly to nitrocellulose membranes. After blocking with $3 \%$ bovine serum albumin, dilutions of mAbs were added. Antigen-antibody reaction was detected by addition of anti-mouse IgG-peroxidase conjugate and the reaction with a chromogenic substrate, 4chloro-1-naphthol $0.05 \%$ in $15 \%$ methanol in the presence of $0.03 \% \mathrm{H}_{2} \mathrm{O}_{2}$.

\section{Results}

\section{Polyclonal species-specific antibodies}

We first tested whether the anti-BthTx-I polyclonal antibodies could give specific antibodies to recognize venoms from $B$. jararacussu and B. jararaca. For this purpose, we produced polyclonal antibodies in rabbits and tested against purified BthTx-I, B. jararacussu and B. jararaca (southeastern) venoms. As expected, the antibodies recognized both venoms when analyzed by ELISA and immunobloting (data not shown). With the goal of eliminating cross-reactive IgGs from polyclonal serum, these antibodies were subjected to affinity chromatography as described in Methods section, which yielded specific IgGs called here species-specific antiBthTx-I. This set of antibodies clearly recognized B. jararacussu venom regardless of whether they were analyzed against individual or pooled venoms or against purified BthTx-I (Fig. 1) and did not recognize B. jararaca venom. These data clearly showed the utility of BthTx-I in generating such specific antibodies.

\section{Production and characterization of monoclonal antibodies (mAbs)}

Since polyclonal antibodies produced against BthTx-I were able to differentiate between the venom of two species, we decided to produce monoclonal antibodies. Fusion of myeloma SP2-O cells with popliteal lymphocytes of mice immunized with BthTx-I resulted in 354 hybridomas of which 21 secreted antibodies against BthTx-I. For cloning, we selected 12 hybridomas, tested by ELISA, that produced the highest optical density $(>1.0)$ of antibodies; these were recloned to ensure monoclonality. Seven stable, immortalized clones secreting anti-BthTx-I antibodies were obtained. These mAbs were designated Bt-1, Bt-2, Bt-3, Bt-6, Bt-10, $\mathrm{Bt}-11$ and Bt-12. All were mAbs belonging to the IgG1 isotype except mAb Bt-6, which belongs to the IgG2b isotype. The mAbs were analyzed by ELISA regarding their ability to recognize venoms from $B$. jararacussu and southeastern B. jararaca. The mAbs Bt-1, Bt-2, Bt-3, Bt-6 and Bt-10 showed specific reactivity for $B$. jararacussu venom while Bt-11 and Bt-12 could recognize both venoms (Fig. 2).

Since ontogenetic and individual variations are described in Bothrops venoms we analyzed the specificity with which mAbs could recognize individual venoms of ten neonates and adults of $B$. jararacussu and southeastern B. jararaca. The mAbs Bt-1, Bt-2, $\mathrm{Bt}-3, \mathrm{Bt}-6$ and Bt-10 were specific for neonates and adults of $B$. jararacussu but did not recognize the venoms of southeastern $B$. jararaca (Fig. 3a). On the other hand, mAbs Bt-11 and Bt-12 recognized both venoms (B. jararacussu and B. jararaca) independently of the age (Fig. 3b).

\section{Analyzing the reactivity of mAbs against the venom of $B$. jararaca from the south of Brazil}

As soon as we discovered the presence of BthTx-I in the venom of $B$. jararaca from the south region of Brazil, we evaluated its reactivity to mAbs using ELISA [15]. First, a serial dilution of mAbs in PBS 


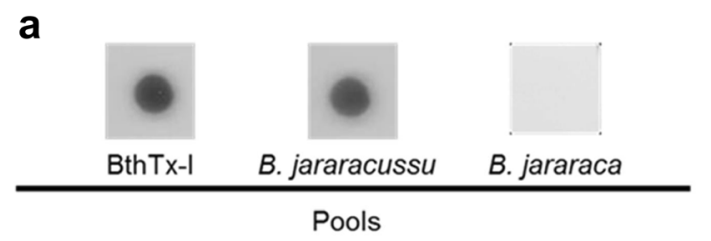

b

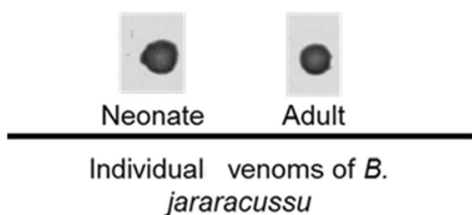

C

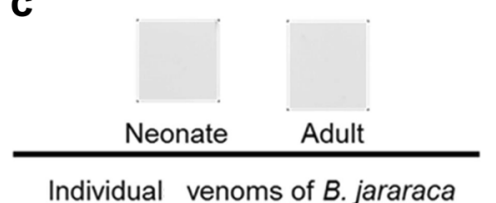

Fig. 1 Species-specific recognition of anti-BthTx-I against B. jararacussu and B. jararaca venom. The purified antibodies were tested against (a) pools, individual venoms of (b) B. jararacussu and (c) southeastern B. jararaca of different ages (neonate and adults) through dot blot. One microgram of each venom was applied to a nitrocellulose membrane and subjected to recognition by the polyclonal species-specific antibodies at a dilution of 1:1000

was added. With a high mAbs concentration, venom of southern B. jararaca could be detected. However, as the mAbs concentration decreased, the intensity of the signal was gradually reduced until complete abrogation at $0.62 \mathrm{ng} / \mu \mathrm{L}$. This result was reproduced with $\mathrm{mAb}$ Bt-1, Bt-2, Bt-3, Bt-6 and Bt-10. Hence, Bt-11 and Bt-12 do not lose reactivity to venom of southern $B$. jararaca, as expected. Figure 4 shows an example of the results obtained using Bt-3 and Bt 11 .

To confirm these data, a dot-blot analysis was carried out in order to compare effects of different mAbs and dilutions against venom from B. jararacussu and southern $B$. jararaca. It was again demonstrated that by using 62 nanograms of mAbs it is possible to detect venom from $B$. jararacussu, but not the one of $B$. jararaca from the south. At that concentration, only Bt-11 and Bt-12 were able to detect BthTx-I in both $B$. jararacussu and B. jararaca venom from the south (Fig. 5).

\section{Cross-reactivity with venoms from different species}

We investigated whether these mAbs would recognize venoms of other Bothrops species from Brazil. The mAbs Bt-1, Bt-2, Bt-6, Bt-10, Bt-11 and Bt-12 showed crossreactivity with the venom of $B$. leucurus, $B$. moojeni, $B$. neuwiedi and $B$. pubescens. However, Bt-3 was highly specific to the venom of $B$. jararacussu, not recognizing any other Brazilian Bothrops venom (Table 1). The mAbs Bt-10 and Bt-11 showed cross-reactivity with the venoms of $B$. diporus and $B$. atrox (Table 1$)$. Dot blot confirmed the results obtained by ELISA (not shown). Monoclonal 3 (Bt-3) showed the highest specificity for $B$. jararacussu venom, highlighting the potential for this antibody as a tool for identifying accidents by $B$. jararacussu in some Brazilian states.

\section{Discussion}

The use of immunodiagnostic tests that allow for the elucidation of the pattern of envenomation caused by

\section{a}

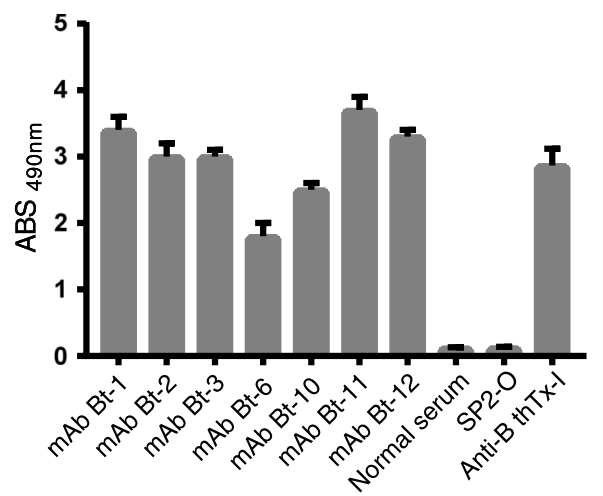

b

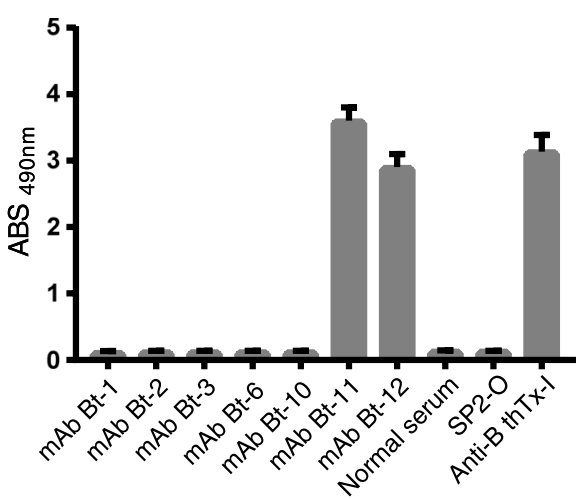

Fig. 2 Analysis of mAb recognition of Bothrops venoms. ELISA plates were sensitized with crude venom of (a) B. jararacussu or (b) southeastern B. jararaca, and then tested with mAbs. The test was performed in triplicate and serum polyclonal anti-BthTx-I produced in mice was used as positive control. Normal serum and supernatant of SP2-O cells provided negative controls 


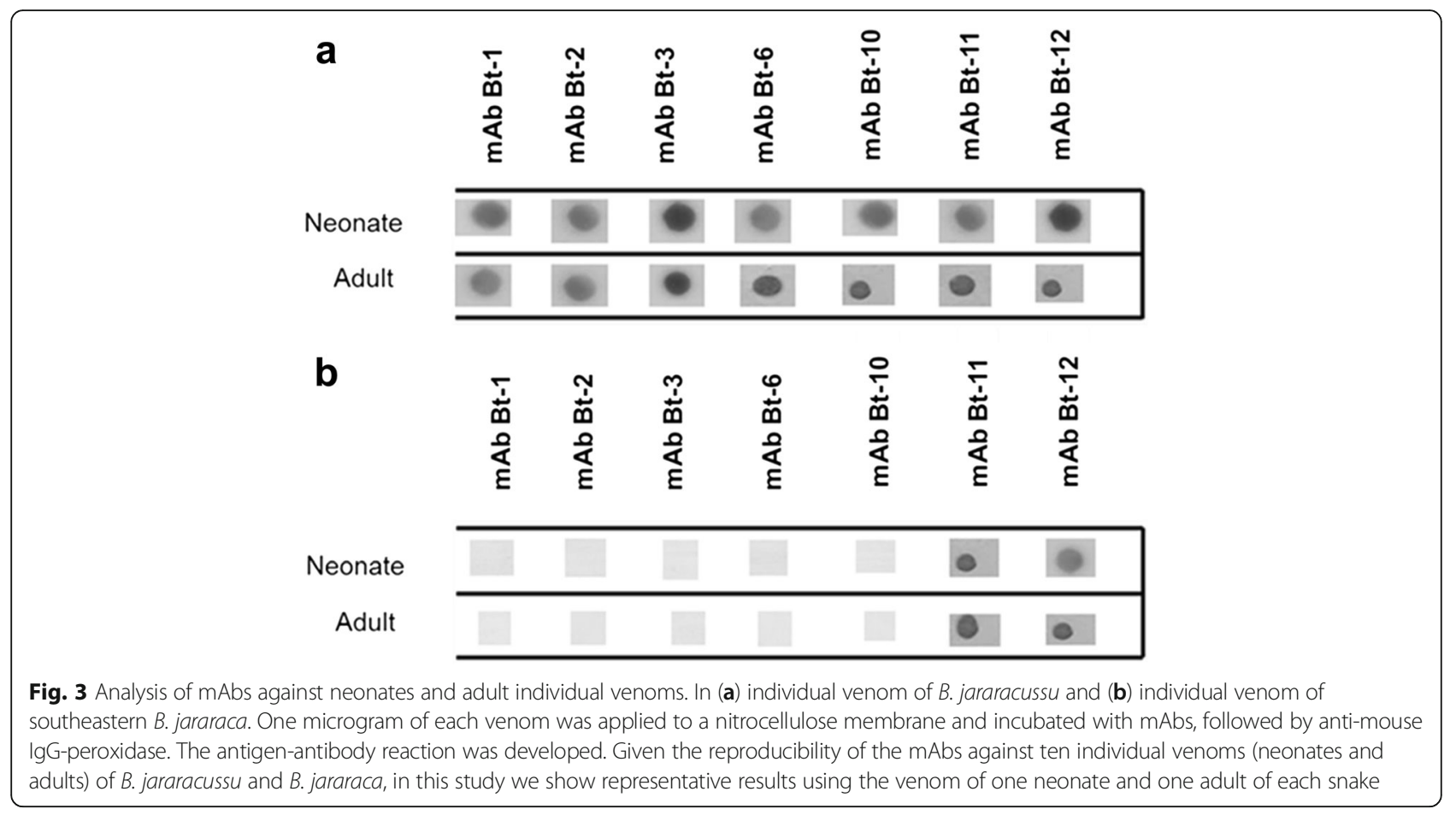

venomous animals is recommended by the World Health Organization. This emphasizes the need to improve the quality of epidemiological and clinical data on accidents caused by venomous animals, in order to improve the therapeutic approach [24]. Nevertheless, common antigens present in venoms from different snake species have shown to be a major problem in developing immunodiagnostic tests [25-27].

In this study, we produced polyclonal antibodies in rabbits against BthTx-I with the aim of differentiating between venoms from $B$. jararacussu and $B$. jararaca, snakes of medical importance for the southeastern region of Brazil, especially the state of Rio de Janeiro. The produced antibodies showed extensive cross-reactivity with $B$. jararaca venom when tested by different immunological methods (data not shown). Then, the cross-reacting molecules were removed by affinity chromatography (Fig. 1). Other authors have successfully used a similar approach to differentiate among the snake venoms from Bothrops, Lachesis, Crotalus and Micrurus due to their overlapping distribution in Brazil [28-31]. However, polyclonal antibodies resulting from immunization of animals vary in different matches, given the variability of animal and immunization protocols [32].

In contrast, the monoclonal antibody constitutes a valuable tool to develop methods for the identification of unknown antigens contained within a mixture of antigens, since each hybridoma is specific for a single antigenic determinant. Because of their high specificity, monoclonal antibodies are standardized reagents that can accurately point out differences in the same or in different molecules, making them important tools in the basic research, immunodiagnosis and clinical studies [33]. Nakamura et al. [34] purified a toxin from Trimeresurus flavoviridis venom (habutobin) and produced monoclonal antibodies that detect habutobin levels in plasma of different animals. Later, Malli and et al. [35] produced monoclonal antibodies against purified toxins from the venom of the spider Cupiennius salei and used it to detect venom in envenomated patients. Such antibodies contributed to an increase in technical specificity of antibodies as immunodiagnostic tools and made it possible to discover the identity of the offending animal [35].

In our study, we showed that monoclonal antibodies raised against BthTx-I are capable of distinguishing between $B$. jararacussu and B. jararaca venoms from different regions, as well as other Bothropic venoms (Table 1). In Brazil, there is no commercial kit available for snake venom detection. Only Australia produces a detection kit, which is based on polyclonal antibodies [36-40]. In this context, the relevance of our work is to generate tools for implementation of a detection kit focused on identifying the offending animal when two species or subspecies of clinical relevance are suspected.

The variability in the composition and activities of snake venoms is reported in several studies and can be seen at various levels including ontogenetic, geographic, sexual, inter and intra-specific [41-44]. In this context, ontogenetic changes in venoms are found in most studies that deal with variability $[45,46]$. Tan et al. [47] 

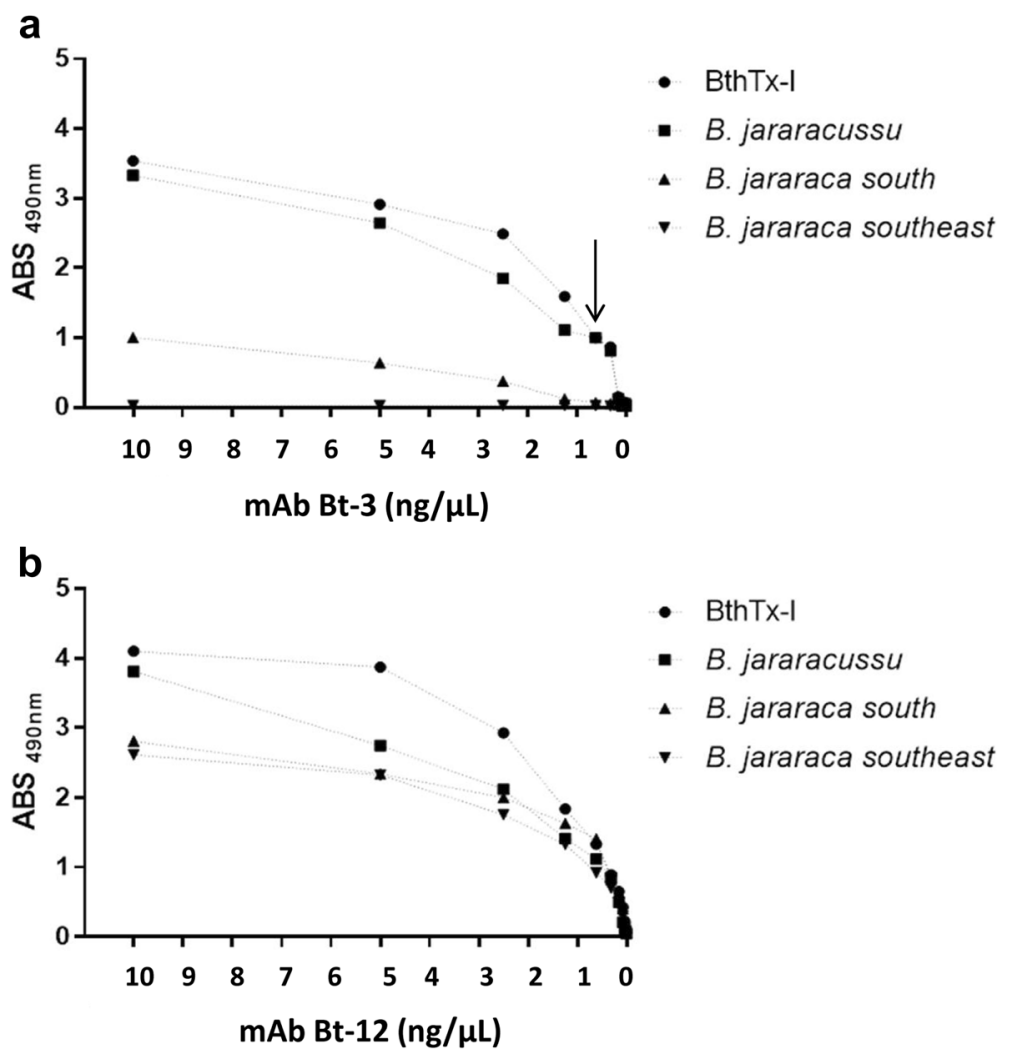

Fig. 4 Titration of monoclonal antibodies. A 96-well plate was sensitized overnight with one microgram of BthTx-I and venoms from B. jararacussu, southeastern B. jararaca and southern B. jararaca. The plate was then incubated with various concentrations of mAb and revealed with anti-mouse lgG labeled with peroxidase. The arrow in (a) shows the concentration of mAb Bt-3 (0.62 ng/ $\mu \mathrm{L})$ which recognizes BthTx-I and B. jararacussu venom. The venoms from $B$. jararaca from the south and southeast were not recognized. In (b), titration of $\mathrm{mAb} B t-12$. Given the reproducibility of the $m A$ Abs against BthTx-I, B. jararacussu, southeastern B. jararaca and southern B. jararaca venoms, only a representative result is showed for mAb Bt-3 (representing results from Bt-1, Bt-2, Bt-6 and Bt-10) and for mAb Bt-12 (representing results from Bt-11)

found qualitative differences in protein profiles of neonates and adults of Notechis scutatus venoms. Furtado et al. [48], analyzing the actions of venoms from nine species of Bothrops snakes, found significant ontogenetic changes in lethal, enzymatic and the blood clotting activities. Our results show that individual B. jararacussu venoms were recognized by monoclonal antibodies, both from adults and neonates. These results show that BthTx-I is present in the venom of B. jararacussu of different ages (Fig. 3). It is also important to note that Lys49-PLA $\mathrm{P}_{2}$ is a conserved toxin present in venoms of many species of Bothrops snakes. Thus, a monoclonal

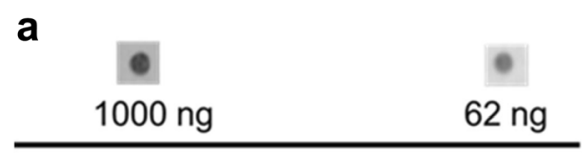

mAb Bt-3

C

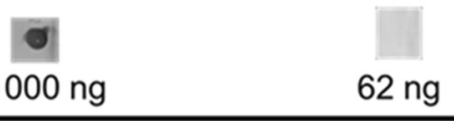

mAb Bt-3 b

$1000 \mathrm{ng} \quad 62 \mathrm{ng}$

mAb Bt-12

d

$\frac{1000 \mathrm{ng}}{\mathrm{mAb} B t-12}$

Fig. 5 Analysis of mAbs against B. jararacussu and B. jararaca (south) venoms. One microgram of B. jararacussu or southern B. jararaca venom was applied directly to a nitrocellulose membrane. After blocking, 1000 or 62 nanograms of mAbs was applied. In (a) and (b) mAbs against B. jararacussu venom and (c) and (d) mAbs against southern B. jararaca venom. Given the reproducibility of the mAbs against the B. jararacussu and southern B. jararaca venoms, only a representative result for $\mathrm{mAb}$ Bt-3 (results from Bt-1, Bt-2, Bt-6 and Bt-10) and another for mAb Bt-12 (results from Bt-11) are showed 
Table 1 Cross-reactivity with Brazilian Bothrops venoms by ELISA

\begin{tabular}{|c|c|c|c|c|c|c|c|c|c|}
\hline & Bt-1 & Bt-2 & Bt-3 & Bt-6 & Bt-10 & Bt-11 & Bt-12 & Anti-BthTx-I & NS \\
\hline \multirow[t]{2}{*}{ Bothrops alternatus } & - & - & - & - & - & - & - & - & - \\
\hline & $(0.134)$ & $(0.110)$ & $(0.136)$ & $(0.112)$ & $(0.103)$ & $(0.141)$ & (0.118) & $(0.151)$ & (0.102) \\
\hline \multirow[t]{2}{*}{ Bothrops atrox } & - & - & - & - & $* * * *$ & $* * * *$ & - & $* * *$ & - \\
\hline & $(0.221)$ & $(0.119)$ & $(0.117)$ & $(0.134)$ & $(2.100)$ & $(2.201)$ & (0.313) & $(2.231)$ & $(0.110)$ \\
\hline \multirow[t]{2}{*}{ Bothrops diporus } & - & - & - & - & - & $* * * *$ & - & $* * * *$ & - \\
\hline & $(0.208)$ & $(0.147)$ & $(0.109)$ & $(0.217)$ & $(0.214)$ & $(2,314)$ & $(0.219)$ & $(2.403)$ & (0.108) \\
\hline \multirow[t]{2}{*}{ Bothrops erytromelas } & - & - & - & - & - & - & - & - & - \\
\hline & $(0.144)$ & $(0.152)$ & $(0.201)$ & $(0.141)$ & $(0.226)$ & $(0.352)$ & $(0.324)$ & $(0.150)$ & (0.104) \\
\hline \multirow[t]{2}{*}{ Bothrops fonsecai } & - & - & - & - & - & - & - & - & - \\
\hline & $(0.187)$ & $(0.138)$ & $(0.184)$ & $(0.209)$ & $(0.213)$ & $(0.247)$ & $(0.298)$ & $(0.162)$ & (0.103) \\
\hline \multirow[t]{2}{*}{ Bothrops insularis } & - & - & - & - & - & - & - & - & - \\
\hline & $(0.201)$ & $(0.192)$ & $(0.199)$ & $(0.229)$ & $(0.114)$ & $(0.232)$ & $(0.288)$ & $(0.142)$ & $(0.111)$ \\
\hline \multirow{2}{*}{$\begin{array}{l}\text { Bothrops jararaca } \\
\text { (southeast) }\end{array}$} & - & - & - & - & - & $* * * *$ & $* * * *$ & $* * *$ & - \\
\hline & $(0.234)$ & $(0.103)$ & $(0.148)$ & $(0.136)$ & $(0.132)$ & $(2.503)$ & (2.603) & (2.633) & $(0.131)$ \\
\hline \multirow[t]{2}{*}{ Bothrops jararacussu } & $* * * *$ & $* * * *$ & $* * * *$ & $* * * *$ & $* * * *$ & $* * * *$ & $* * * *$ & $* * * *$ & - \\
\hline & $(2.657)$ & $(2.421)$ & $(2.303)$ & $(2.529)$ & $(2.437)$ & (2.509) & (2.619) & $(2.628)$ & $(0.122)$ \\
\hline \multirow[t]{2}{*}{ Bothrops leucurus } & $* * * *$ & $* *$ & - & $* * * *$ & $* * * *$ & $* * * *$ & - & **** & - \\
\hline & $(1.407)$ & $(0.634)$ & $(0.132)$ & $(1.914)$ & (2.098) & $(2.001)$ & $(0.215)$ & $(2.383)$ & $(0.134)$ \\
\hline \multirow[t]{2}{*}{ Bothrops moojeni } & $* * * *$ & $* *$ & - & $* * * *$ & $* * * *$ & $* * * *$ & $* * * *$ & $* * * *$ & - \\
\hline & $(1.543)$ & $(0.899)$ & $(0.131)$ & $(2.008)$ & (2.103) & $(2.125)$ & $(2.244)$ & (2.479) & $(0.143)$ \\
\hline \multirow[t]{2}{*}{ Bothrops neuwiedi } & $* * * *$ & $* *$ & - & $* * * *$ & $* * * *$ & $* * * *$ & - & $* * * *$ & - \\
\hline & $(1.722)$ & $(0.987)$ & $(0.124)$ & $(2.103)$ & $(2.103)$ & $(2.136)$ & $(0.200)$ & $(2.501)$ & $(0.127)$ \\
\hline \multirow[t]{2}{*}{ Bothrops pubescens } & $* * * *$ & $* *$ & - & $* * * *$ & $* * * *$ & $* * * *$ & - & $* * * *$ & - \\
\hline & $(1.481)$ & $(0.923)$ & $(0.116)$ & $(2.002)$ & $(2.103)$ & $(2.212)$ & $(0.235)$ & $(2.426)$ & $(0.149)$ \\
\hline
\end{tabular}

Polyclonal anti-BthTx-I serum and normal serum (NS) of mice were utilized

**** Strong binding (optical density > 1.0); ** medium binding (optical density between 0.5 and 1.0 ); - weak or no binding (optical density between 0.1 and 0.5 )

antibody that recognizes BthTx-I may recognize the homologous molecules in other venoms, explaining our data in the ELISA assay (Table 1).

A similar profile of recognition can be observed when comparing our monoclonal antibodies with those described by Prado et al. [49]. The camelid antibody fragments (VHH) with specificity for BthTx-I and BthTx-II from $B$. jararacussu venom were selected from an immune $\mathrm{VHH}$ phage display library. Corroborating our results, some clones showed reactivity against venoms from B. moojeni, B. leucurus and B. diporus and did not recognize $B$. alternatus venom [49]. This last venom contains a protein with $90 \%$ homology with BthTX-I, called BaTX. Ponce-Soto et al. [18] isolated and characterized BaTX that constitutes approximately $5 \%$ of the crude venom. We suggest that this low level of BaTX in $B$. alternatus venom is the cause of the lack of recognition.

In this context, BthTx-I from the southern $B$. jararaca venom shows $100 \%$ homology with BthTx-I from $B$. jararacussu venom, and it represents $13.8 \%$ of the proteins in southern $B$. jararaca venom [19]. This could be an obstacle to spreading the use of monoclonal antibodies developed by our group to differentiate among $B$. jararaca bites. However, the BthTx-I from B. jararacussu venom represents approximately $40 \%$ of total proteins, the highest concentration described so far for Bothrops venoms $[12,13]$. This difference in BthTx-I concentration between the two Bothrops species may influence the signal generated by mAbs in ELISA. For this reason, the dilution of antibodies preserves only $B$. jararacussu venom detection, since it is the venom with the higher level of BthTx-I among all Bothrops species (Figs. 4 and 5). Furthermore, accidents caused by $B$. jararacussu and B. jararaca are epidemiologically relevant in Rio de Janeiro State [3], where B. jararaca do not produce BThx-I. Thus, the use of a diagnostic kit from a $\mathrm{mAb}$ may be regionally important. A diagnostic kit able to discriminate between $B$. jararaca and $B$. jararacussu venoms would solve, in Rio de Janeiro, questions related to epidemiology and treatment of patients.

There is a growing interest in the standardization of the ELISA technique in the form of an immunodiagnostic 
test, as used in Australia. It could be used in different locations and could offer to healthcare professionals a quick diagnosis of the genus involved in the accident and the amount of injected venom. Moreover, it is noteworthy that the data on snakebite are quite fragmentary because of the lack of information and recorded data. Therefore, the development of a regional diagnostic kit for identification of snake venoms could improve the treatment of snakebite victims and contribute to epidemiological studies.

\section{Conclusion}

Our results demonstrate the potential of BthTx-I as a biomarker molecule to be used for antibody production (monoclonal and polyclonal) and posterior specific detection of $B$. jararacussu venom. These antibodies may be an important tool for the development of a detection kit that differentiates between venoms from $B$. jararacussu and B. jararaca, especially in Rio de Janeiro where both species are epidemiologically relevant.

\section{Abbreviations}

BthTx-I: Bothropstoxin-l; mAbs: monoclonal antibodies

\section{Acknowledgments}

The authors would like to thank Ana Lúcia de Oliveira Carvalho and Dione Maria da Silva for their technical assistance and Dr. Martha Sorenson for English review. Thanks are also due to the Center for the Study of Venoms and Venomous Animals (CEVAP) of UNESP for enabling the publication of this paper (Edital Toxinologia CAPES no. 063/2010, Process no. 230.38.006285/2011-21, AUXPE Toxinologia 1219/2011).

\section{Funding}

The present work was supported by the National Council for Scientific and Technological Development (CNPq), Rio de Janeiro Research Foundation (FAPERJ) and by by the Edital Toxinologia CAPES no. 063/2010, Process no. 2308.006277/2011-85, AUXPE 1517/2011.

\section{Authors' contributions}

RTA wrote the article draft, performed the data collection, analysis and interpretetion. CCN participate in experimental design edited and revised the article. LBM and CRFS participated in data collection and critical revision of the article. IF supervised the experiments on monoclonal antibodies and performed a critical revision of the article. RBZ supervised the experiments, contributed to development of concept and experimental design, writing, reviewing and editing the article. All authors read and approved the final manuscript.

\section{Competing interests}

The authors declare that they have no competing interests.

\section{Consent for publication}

Not applicable.

\section{Ethics approval and consent to participate}

All animal procedures were approved by the Ethics Committee for Animal Research of Butantan Institute (process no. 697/10).

\section{Author details}

${ }^{1}$ Laboratório de Hemostase e Venenos, Instituto de Bioquímica Médica Leopoldo de Meis, Universidade Federal do Rio de Janeiro (UFRJ), Rio de Janeiro, RJ, Brasil. ${ }^{2}$ Instituto Vital Brazil, Niterói, RJ, Brasil. ${ }^{3}$ Instituto Butantan, São Paulo, SP, Brasil.
Received: 22 September 2016 Accepted: 24 February 2017

Published online: 11 March 2017

\section{References}

1. dos Santos MC, Gonçalves LRC, Fortes-Dias CL, Cury Y, Gutiérrez JM, Furtado MFD. A eficácia do antiveneno botrópico-crotálico na neutralização das principais atividades do veneno de Bothrops jararacussu. Rev Inst Med Trop São Paulo. 1992;34(2):77-83.

2. Luna KPO, da Silva MB, Pereira VRA. Clinical and immunological aspects of envenomations by Bothrops snakes. J Venomous Anim Toxins Incl Trop Dis. 2011;17(2):130-41

3. Melgarejo AR. Serpentes peçonhentas do Brasil. In: Cardoso JLC, França FOS, Fan HW, Málaque CMS, Haddad Jr, editors. Animais peçonhentos no Brasil: Biologia, clínica e terapêutica dos acidentes. São Paulo: Sarvier; 2003. p. 33-61.

4. Maruyama M, Kamiguti AS, Cardoso JLC, Sano-Martins IS, Chudzinski AM, Santoro ML, et al. Studies on blood coagulation and fibrinolysis in patients bitten by Bothrops jararaca (jararaca). Thromb Haemost. 1990;63(3):449-53.

5. Sanchez EF, Freitas TV, Ferreira-Alves DL, Velarde DT, Diniz MR, Cordeiro MN, et al. Biological activities of venoms from South American snakes. Toxicon. 1992:30(1):95-103.

6. Brazil V. A defesa contra o ofidismo. São Paulo: Pocai \& Weiss; 1911

7. Dias da Silva W, Guidolin R, Raw I, Higashi HG, Morais CP, Lima JF, et al. Cross-reactivity of horse monovalent antivenoms to venoms of ten Bothrops species. Mem Inst Butantan. 1989;51(4):153-68.

8. Oshima-Franco Y, Leite GB, Silva GH, Cardoso DF, Hyslop S, Giglio JR, et al. Neutralization of the pharmacological effects of bothropstoxin-I from Bothrops jararacussu (jararacuçu) venom by crotoxin antiserum and heparin. Toxicon. 2001;39(10):1477-85.

9. Zamunér SR, da Cruz-Höfling MA, Corrado AP, Hyslop S, Rodrigues-Simioni L. Comparison of the neurotoxic and myotoxic effects of Brazilian Bothrops venoms and their neutralization by commercial antivenom. Toxicon. 2004:44(3):259-71.

10. Beghini DG, da Cruz-Höfling MA, Randazzo-Moura P, Rodrigues-Simioni L, Novello JC, Hyslop S, et al. Cross-neutralization of the neurotoxicity of Crotalus durissus terrificus and Bothrops jararacussu venoms by antisera against crotoxin and phospholipase $\mathrm{A}_{2}$ from Crotalus durissus cascavella venom. Toxicon. 2005;46(6):604-11.

11. Correa-Netto C, Teixeira-Araujo R, Aguiar AS, Melgarejo AR, De-Simone SG, Soares MR, et al. Immunome and venome of Bothrops jararacussu: a proteomic approach to study the molecular immunology of snake toxins. Toxicon. 2010;55(7):1222-35.

12. Kashima S, Roberto PG, Soares AM, Astolfi-Filho S, Pereira JO, Giuliati S, et al. Analysis of Bothrops jararacussu venomous gland transcriptome focusing on structural and functional aspects: I-gene expression profile of highly expressed phospholipases A2. Biochimie. 2004;86(3):211-9.

13. Homsi-Brandeburgo MI, Queiroz LS, Santo-Neto H, Rodrigues-Simioni CL, Giglio JR. Fractionation of Bothtrops jararacussu snake venom: partial chemical characterization and biological activity of bothropstoxin. Toxicon. 1988;26(7):473-9.

14. Rodrigues-Simioni L, Borgese N, Ceccarelli B. The effects of Bothrops jararacussu venom and its components on frog nerve-muscle preparation. Neuroscience. 1983;10(2):475-89.

15. Lomonte B, Angulo Y, Calderón L. An overview of lysine-49 phospholipase A2 myotoxins from crotalid snake venoms and their structural determinants of myotoxic action. Toxicon. 2003;42(8):885-901.

16. Soares AM, Guerra-Sá R, Borja-Oliveira CR, Rodrigues VM, Rodrigues-Simioni $L$, Rodrigues $V$, et al. Structural and functional characterization of BnSP-7, a Lys49 myotoxic phospholipase A(2) homologue from Bothrops neuwiedi pauloensis venom. Arch Biochem Biophys. 2000;378(2):201-9.

17. Soares AM, Andrião-Escarso SH, Angulo Y, Lomonte B, Gutiérrez JM, Marangoni $S$, et al. Structural and functional characterization of myotoxin I, a Lys49 phospholipase A(2) homologue from Bothrops moojeni (Caissaca) snake venom. Arch Biochem Biophys. 2000;373(1):7-15.

18. Ponce-Soto LA, Lomonte B, Gutiérrez JM, Rodrigues-Simioni L, Novello JC, Marangoni S. Structural and functional properties of BaTX, a new Lys49 phospholipase A2 homologue isolated from the venom of the snake Bothrops alternatus. Biochim Biophys Acta. 2007;1770(4):585-93.

19. Gonçalves-Machado L, Pla D, Sanz L, Jorge RJ, Leitão-De-Araújo M, Alves ML, et al. Combined venomics, venom gland transcriptomics, bioactivities, and antivenomics of two Bothrops jararaca populations from geographic isolated regions within the Brazilian Atlantic rainforest. J Proteome. 2016;135:73-89. 
20. Fernandes I, Assumpção GG, Silveira CR, Faquim-Mauro EL, Tanjoni I, Carmona AK, et al. Immunochemical and biological characterization of monoclonal antibodies against BaP1, a metalloproteinase from Bothrops asper snake venom. Toxicon. 2010;56(6):1059-65.

21. Köhler G, Milstein C. Continuous cultures of fused cells secreting antibody of predefined specificity. Nature. 1975;256(5517):495-7.

22. Theakston RDG, Lloyd-Jones MJ, Reid HA. Micro-ELISA for detecting and assaying snake venom and venom antibody. Lancet. 1977;2(8039):639-41.

23. Towbin H, Staehelin T, Gordon J. Electrophoretic transfer of proteins from polyacrylamide gels to nitrocellulose sheets: procedure and some applications. Proc Natl Acad Sci. 1979;76(9):4350-4.

24. World Health Organization. Progress in the characterization of venoms and standardization of antivenoms. WHO Offset Public. 1981;(58):1-44. http://www.who.int/iris/handle/10665/37282.

25. McCarthy NJ. Snake venom detection kit. Med J Aust. 1984;140(9):518.

26. Marshall LR, Herrmann RP. Cross-reactivity of bardick snake venom with death adder antivenom. Med J Aust. 1984;140(9):541-2.

27. Ho M, Warrell MJ, Warrell DA, Bidwell D, Voller A. A critical reappraisal of the use of enzyme-linked immunosorbent assays in the study of snake bite. Toxicon. 1986;24(3):211-21.

28. Heneine LG, Catty D, Theakston RDG. Development of a species-specific ELISA for Brazilian pit-viper venoms. Braz J Med Biol Res. 1990;23(6-7):585-8.

29. Audebert F, Grosselet O, Sabouraud A, Bon C. Quantitation of venom antigens from European vipers in human serum or urine by ELISA.J Anal Toxicol. 1993;17(4):236-40.

30. Heneine LG, Catty D. Species-specific detection of venom antigens from snakes of the Bothrops and Lachesis genera. Toxicon. 1993;31(5):591-603.

31. Chavez-Olortegui C, Lopes CS, Cordeiro FD, Granier C, Diniz CR. An enzyme linked immunosorbent assay (ELISA) that discriminates between Bothrops atrox and Lachesis muta muta venoms. Toxicon. 1993;31(4):417-25.

32. Nelson PN, Fletcher SM, MacDonald D, Goodall DM, Jefferis R. Assay restriction profiles of three monoclonal antibodies recognizing the $\mathrm{G} 3 \mathrm{~m}(\mathrm{u})$ allotype. Development of an allotype specific assay. J Immunol Methods. 1991;138(1):57-64

33. Blottière HM, Daculsi G, Anegon I, Pouezat JA, Nelson PN, Passuti N. Utilization of activated U937 monocytic cells as a model to evaluate biocompatibility and biodegradation of synthetic calcium phosphate. Biomaterials. 1995;16(6):497-503.

34. Nakamura M, Hanashiro K, Kosugi T. A modification of the ELISA-double sandwich method for estimating the concentration of habutobin. Toxicon. 1993;31(10):1325-8.

35. Malli H, Imboden H, Kuhn-Nentwig L. Quantifying the venom dose of the spider Cupiennius salie using monoclonal antibodies. Toxicon. 1998;36(12):1959-69.

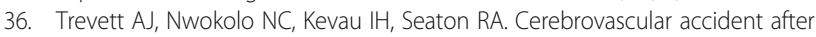
taipan bite. Med J Aust. 1994;160(2):94.

37. Sutherland SK, Leonard RL. Snakebite deaths in Australia 1992-1994 and a management update. Med J Aust. 1995;163(11-12):616-8.

38. Trevett AJ, Lalloo DG, Nwokolo NC, Theakston DG, Naraqi S, Warrell DA. Venom detection kits in the management of snakebite in Central province, Papua New Guinea. Toxicon. 1995;33(5):703-5.

39. Mead HJ, Jelinek GA. Suspected snakebite in children: a study of 156 patients over 10 years. Med J Aust. 1996;164(8):467-70.

40. Southern DA, Callanan VI, Gordon GS. Severe envenomation by the taipan (Oxyuranus scutellatus). Med J Aust. 1996;165(11-12):662-4.

41. Chippaux JP, Willians V, White J. Snake venoms variability: methods of study, results and interpretation. Toxicon. 1991;29(11):1279-303.

42. Martins AM, Toyama MH, Havt A, Novello JC, Marangoni S, Fonteles MC, et al. Determination of Crotalus durissus cascavella venom components that induce renal toxicity in isolated rat kidneys. Toxicon. 2002;40(8):1165-71.

43. Mushinsky HR, Miller DE. Predation on Water Snakes: Ontogenetic and Interspecific Considerations. Copeia. 1993;1993(3):660-5.

44. Andrade RO, Silvano RAM. Comportamento alimentar e dieta da "falsa coral" Oxyrhopus guibei Hoge \& Romano (Serpentes, Colubridae). Rev Bras Zool. 1996;13(1):143-50.

45. López-Lozano JL, de Sousa MV, Chávez-Olortegui C, Sanchez EF, Muniz EG, Bührnheim PF, et al. Ontogenetic variation of metalloproteinases and plasma coagulant activity in venoms of wild Bothrops atrox specimens from Amazonian rain forest. Toxicon. 2002;40(7):997-1006.

46. Saldarriaga MM, Otero R, Núñez V, Toro MF, Díaz A, Gutiérrez JM. Ontogenetic variability of Bothrops atrox and Bothrops asper snake venoms from Colombia. Toxicon. 2003;42(4):405-11.
47. Tan NH, Ponnudurai G, Mirtschin PJ. A comparative study on the biological properties of venoms from juvenile and adult common tiger snake (Notechis scutatus) venoms. Comp Biochem Physiol B. 1993;106(3):651-4.

48. Furtado MF, Maruyama M, Kamiguti AS, Antonio LC. Comparative study of nine Bothrops snake venoms from adult female snakes and their offspring. Toxicon. 1991;29(2):219-26.

49. Prado ND, Pereira SS, da Silva MP, Morais MS, Kayano AM, Moreira-Dill LS, et al. Inhibition of the Myotoxicity Induced by Bothrops jararacussu Venom and Isolated Phospholipases A2 by Specific Camelid Single-Domain Antibody Fragments. PLoS One. 2016;11(3):e0151363.

\section{Submit your next manuscript to BioMed Central and we will help you at every step:}

- We accept pre-submission inquiries

- Our selector tool helps you to find the most relevant journal

- We provide round the clock customer support

- Convenient online submission

- Thorough peer review

- Inclusion in PubMed and all major indexing services

- Maximum visibility for your research

Submit your manuscript at www.biomedcentral.com/submit
Biomed Central 